\title{
Cytoplasmic expression of SOX9 as a poor prognostic factor for oral squamous cell carcinoma
}

\author{
YOSHIMASA SUMITA $^{1,2}$, MANABU YAMAZAKI ${ }^{1}$, SATOSHI MARUYAMA $^{3}$, TATSUYA ABÉ ${ }^{4}$, \\ JUN CHENG $^{1}$, RITSUO TAKAGI ${ }^{2}$ and JUN-ICHI TANUMA ${ }^{1}$ \\ Divisions of ${ }^{1}$ Oral Pathology and ${ }^{2}$ Oral and Maxillofacial Surgery, \\ Niigata University Graduate School of Medical and Dental Sciences, Niigata 951-8514; \\ ${ }^{3}$ Oral Pathology Section, Department of Surgical Pathology, Niigata University Hospital, Niigata 951-8520; \\ ${ }^{4}$ Division of Molecular and Diagnostic Pathology, Niigata University Graduate School of \\ Medical and Dental Sciences, Niigata 951-8122, Japan
}

Received March 19, 2018; Accepted August 7, 2018

DOI: 10.3892/or.2018.6665

\begin{abstract}
Transcription factor SRY-box 9 (SOX9) is a key regulator of chondrocyte differentiation and sex determination, and it is also involved in the progression of various types of human cancer. However, its putative association with oral squamous cell carcinoma (OSCC) remains elusive. The aim of the present study was to investigate the expression profiles of SOX9 in various oral epithelial lesions, including OSCC. We performed immunohistochemical analysis of SOX9 expression in surgical specimens of OSCC, which simultaneously exhibited different grades of epithelial lesions, and analyzed the correlation between SOX9 expression and several clinicopathological factors. Moreover, we performed immunofluorescent staining, western blot analysis and real-time reverse transcription-polymerase chain reaction to assess SOX9 expression in OSCC HSC-3 (a metastatic cell line) and HSC-4 (a non-metastatic cell line) cell lines. In surgical specimens, SOX9 expression was detected in the nuclei of proliferating cells in areas with epithelial dysplasia and carcinoma in situ, but not in areas with normal epithelia. Nuclear SOX9 expression was observed in most SCC cells. Notably, cytoplasmic SOX9 expression was confirmed only in some SCC cells; however, cytoplasmic SOX9 expression was significantly and positively correlated with poor clinical outcomes. Both protein and mRNA expression of SOX9 were significantly higher in the HSC-3 cell line than that in the HSC-4 line. Notably, however, only HSC-3 cells exhibited cytoplasmic localization of SOX9 expression. Our findings
\end{abstract}

Correspondence to: Dr Manabu Yamazaki, Division of Oral Pathology, Niigata University Graduate School of Medical and Dental Sciences, 2-5274 Gakkocho-dori, Chuo-ku, Niigata 951-8514, Japan

E-mail: manyamaz@dent.niigata-u.ac.jp

Key words: SOX9, oral mucosa, squamous cell carcinoma, prognosis, intracellular expression patterns indicate that SOX9 may be involved in the tumorigenesis and progression of OSCC. Furthermore, its cytoplasmic expression represents a potential predictive biomarker for tumor aggressiveness and OSCC prognosis.

\section{Introduction}

Sex-determining region Y (SRY)-box 9 (SOX9) is a member of a transcription factor family characterized by similarities in the high-mobility group DNA-binding domain of SRY (1). It plays crucial roles in the development and differentiation of diverse tissues, such as cartilage $(1,2)$ and testes (3). During chondrogenesis, SOX9 transactivates the genes encoding extracellular matrix (ECM) proteins, including type II collagen and aggrecan, which function as a foothold for chondrocytes (4). During male gonadogenesis, SOX9 specifies the Sertoli cell lineage and upregulates the transcription of a gene encoding anti-Müllerian hormone, which inhibits the development of the female gonads (3). SOX9 is also crucial for the development and maintenance of ectodermal tissues. In adult skin, SOX9 is expressed in the sebaceous glands, sweat glands, outer root sheath of the hair follicles (5), and basal layer of the human epidermis, where it acts as an important regulator of epidermis and cutaneous appendages through pro-proliferative and anti-apoptotic activities (6).

Recently, SOX9 was shown to be involved in the initiation and/or progression of various types of cancers, including prostate (7), breast (8) and colorectal (9) cancers, basal cell carcinoma of the skin (10) and esophageal squamous cell carcinoma (SCC) (11). These studies have described higher SOX9 expression levels in cancer cells than in their normotypic counterparts (7-11). In addition, three of the studies have reported that high SOX9 expression levels in cancer cells are positively correlated with poor clinical outcomes $(8,9,11)$. However, in oral squamous cell carcinoma (OSCC), both the expression profile of SOX9 and its possible clinicopathological correlates remain unknown.

We previously described the importance of ECM proteins in the pathogenesis of OSCC $(12,13)$ and salivary gland 
tumors $(14,15)$. We demonstrated that the keratinocyte-derived, basement membrane-type heparan sulfate proteoglycan perlecan is deposited in the intraepithelial spaces during oral epithelial carcinogenesis (12). Indeed, we revealed that perlecan was abundantly expressed around the invasive nests of OSCC and that this increased expression in the stromal space was a useful indicator of stromal invasion (13). Furthermore, perlecan acts as a reservoir for growth factors, such as keratinocyte growth factor (14) and vascular endothelial growth factor (16). Perlecan expression is regulated by the $N F-\kappa B$ pathway, which regulates the transcription of the perlecan-encoding HSPG 2 gene in the microenvironment of prostate cancer (17). Transcriptional profiling of oncogene-expressing cells with a SOX9 deletion has demonstrated that SOX 9 is a potent positive regulator of ECM (including perlecan) gene transcription (18).

Given the above reports, we aimed to characterize the immunohistochemical expression profiles of SOX9 in surgical samples of oral mucosa that contained foci of epithelial dysplasia, carcinoma in situ (CIS), and SCC as well as in cultured cells of OSCC origin. In addition, we investigated the use of SOX9 expression as a novel prognostic marker for OSCC.

\section{Materials and methods}

Tissue samples. Forty-nine consecutive patients (27 males and 22 females) who underwent primary surgical treatment for OSCC from January 2008 to December 2011 were included in the present study. Patients with recurrent tumors were excluded. The follow-up clinical information was obtained from the patient medical records. The follow-up period was measured from the date of surgery until death or until the final follow-up record. Clinical stage was determined according to the Union for International Cancer Control TNM classification system, 7th edition (19). The median age of the patients at surgery was 69 years (range, 22-88 years).

Surgical specimens were fixed in $10 \%$ formalin and embedded in paraffin. After examining hematoxylin and eosin (H\&E)-stained sections from each specimen, the paraffin block from each patient that simultaneously contained (maximum possible) the SCC area, including the invasive front, and areas of adjacent epithelia appearing normal, dysplastic, or as CIS was selected. Serial $2.5-\mu$ m-thick sections were prepared for H\&E staining and immunohistochemistry. In addition, samples of metastatic lymph nodes were collected from 17 of the 19 patients with late metastasis in the cervical lymph nodes. The Ethics Board of the Niigata University Graduate School of Medicine and Dental Sciences (Oral Life Science) reviewed and approved the experimental protocol for analyzing the surgical materials (approval no. 12-10-13) and written informed consent was obtained from the patients.

Antibodies. The following antibodies were used for immunostaining: Anti-SOX9 (1:1,500; rabbit polyclonal; cat. no. HPA001758; Atlas Antibodies, Stockholm, Sweden); anti-perlecan (1:6,000; rabbit polyclonal) was established by Saku and Furthmayr as previously described (20); anti-keratin 17 (K17) (1:100; mouse monoclonal; cat. no. M7046; clone E3; Dako, Glostrup, Denmark); and anti-Ki-67 (1:100; mouse monoclonal; cat. no. M7240; clone MIB-1; Dako). For western blotting, a different anti-SOX9 antibody (1:1,000; rabbit monoclonal; cat. no. 82630; clone D8G8H; Cell Signaling Technology, Inc., Danvers, MA, USA) was used.

Immunohistochemistry. Immunohistochemical analysis of paraffin sections was performed using the ChemMate EnVision system (Dako) according to the manufacturer's instructions. Deparaffinized and rehydrated sections were immersed in $0.3 \%$ hydrogen peroxide in methanol for $30 \mathrm{~min}$ at room temperature to block endogenous peroxidase activities. For SOX9, K17 and Ki-67 immunostaining, antigen retrieval was performed by first autoclaving sections in citric acid buffer (pH 6.0) at $121^{\circ} \mathrm{C}$ for $10 \mathrm{~min}$. To analyze the stromal expression of perlecan, the sections were incubated with hyaluronidase (Type IS, 330 UI/ml; Sigma-Aldrich; Merck KGaA, Darmstadt, Germany) for 30 min prior to immunostaining. After antigen retrieval, the sections were incubated with 5\% milk protein in phosphate-buffered saline (PBS) for $30 \mathrm{~min}$ and then with the primary antibodies overnight at $4^{\circ} \mathrm{C}$. After washing with PBS, the sections were reacted with EnVision (Dako) for $60 \mathrm{~min}$ at room temperature. Peroxidase reaction products were developed with 3,3'-diaminobenzidine, and the sections were counterstained with hematoxylin. In the control experiments, the primary antibodies were replaced with the appropriate negative control immunoglobulin (cat. no. X0931 for mouse IgG1 and cat. no. X0903 for rabbit Ig fraction; Dako).

SOX9 expression. To investigate the localization of SOX9 in squamous cell epithelial lesions, the combined H\&E and immunohistochemical findings were used to first define the foci on a given section on a case-by-case basis, which demonstrated normal epithelia, epithelial dysplasia, and CIS, if present. Nuclear and cytoplasmic positivity was evaluated individually for foci of each type of epithelia and for SCC; foci were positive if they occupied $\geq 10 \%$ of the area of stained cells. For the SCC components, the proportion of the entire area of the SCC demonstrating SOX9-positive cancer cells was manually semi-quantified on the representative sections of each specimen.

Cell lines. Human cell lines derived from OSCC HSC-3 and HSC-4 cells were obtained from Riken BRC Cell Bank (Tsukuba, Japan). To analyze the association between SOX9 expression and metastasis, two cell lines, namely HSC-3, a metastatic cell line that was established from the metastatic lymph node of a 63-year-old man with poorly differentiated SCC and HSC-4, a non-metastatic cell line that was established from a metastatic lymph node in a 63-year-old man with well-differentiated SCC, were selected (21). The cells were maintained in Minimum Essential Medium (Thermo Fisher Scientific, Inc., Waltham, MA, USA) supplemented with $10 \%$ fetal calf serum (FCS) under a humidified atmosphere with $5 \% \mathrm{CO}_{2}$ and $95 \%$ air at $37^{\circ} \mathrm{C}$.

Immunofluorescence. The HSC-3 and HSC-4 cells were seeded on a coverslip in $35-\mathrm{mm}$ dishes at a cell concentration of $0.25 \times 10^{5}$ cells/dish. After 3 days, the cells were washed with PBS and fixed with $4 \%$ paraformaldehyde for $30 \mathrm{~min}$ at room temperature. Then, the cells were permeabilized with $0.2 \%$ Triton X-100 in PBS, and then incubated with 5\% milk protein 
in PBS at $4^{\circ} \mathrm{C}$ overnight to block non-specific binding. The cells were incubated with the anti-SOX 9 primary antibody for 60 min. After washing with PBS, the cells were reacted with Alexa Fluor ${ }^{\mathrm{TM}} 568$ goat anti-rabbit IgG secondary antibody (1:200; cat. no. A11011; Thermo Fisher Scientific, Inc.). The coverslips were mounted with ImmunoSelect Antifading Mounting Medium DAPI (Dianova, Hamburg, Germany), and the cells were observed using a Nikon Eclipse E600W immunofluorescence microscope (Nikon Corp., Tokyo, Japan). The fluorescence intensities for SOX9 and DAPI staining were measured using the NIS-Elements AR software (Nikon Corp.).

Scratch wound-healing assay. Artificial wounds were inflicted on the confluent monolayers of HSC-3 and HSC-4 cells in 60-mm culture dishes using a $200-\mu 1$ pipette tip. Every $6 \mathrm{~h}$ for $36 \mathrm{~h}$, the process of migration into the wound areas was monitored under a phase-contrast microscope equipped with a digital camera. Wound surface area was measured on the captured images of four fields using ImageJ (version 1.50i; National Institutes of Health, Bethesda, MD, USA). Percent (\%) closure of the wound surface area was calculated as open surface area/total surface area (surface area at $0 \mathrm{~h}$ ) x 100.

Growth curve analysis. The cells were seeded at a concentration of $0.25 \times 10^{5}$ cells in $60-\mathrm{mm}^{2}$ dishes and incubated at $37^{\circ} \mathrm{C}$ for 6 days. Thereafter, the cells were counted on days 2 , 4 and 6 . The average number of proliferating cells was calculated from triplicate experiments.

Real-time reverse transcription-polymerase chain reaction (RT-PCR). HSC-3 and HSC-4 cells were lysed with ISOGEN (Nippon Gene Co., Ltd., Tokyo, Japan) for the extraction of total RNA. cDNA was synthesized from each $5-\mu \mathrm{g}$ RNA sample using the SuperScript III First-Strand Synthesis system (Thermo Fisher Scientific, Inc.), followed by PCR. RT-PCR was performed with specific primers for SOX9 using a MiniOpticon Real-Time PCR Detection system CFB-3120 (Bio-Rad Laboratories, Inc., Hercules, CA, USA). Gene expression levels were quantified by the $\Delta \Delta \mathrm{Cq}$ method (22) using CFX Manager Software version 2.1 (Bio-Rad Laboratories). The expression of the peptidylprolyl isomerase A (PPIA) gene was used to normalize for variance, and the expression levels of specific genes were represented as ratios relative to the expression level of PPIA from the same master reaction. PCR primer pairs used for each gene were: 5'-AGCTCTGGAGAC TTCTGAACGAGAG-3' and 5'-CGTTCTTCACCGACTTCC TCC-3' for SOX9; and 5'-GCAGTAATGGGTTACTTCTGA AAC-3' and 5'-TGCCTCAGGTAATACATTACAGAC-3' for PPIA.

Western blotting. For the extraction of total protein, the HSC-3 and HSC-4 cells were lysed at day 5 after seeding with M-PER ${ }^{\mathrm{TM}}$ Mammalian Protein Extraction reagent (Thermo Fisher Scientific, Inc.). Thereafter, the cell lysates were used for western blotting as described elsewhere (23). Primary antibody for SOX9 was used at a dilution of 1:1,000. Labeled protein bands were visualized using the ECL Prime Western Blotting Detection system (GE Healthcare UK, Ltd., Little Chalfont, UK).
Table I. SOX9 expression profiles in oral epithelial lesions.

\begin{tabular}{lccc}
\hline & & \multicolumn{2}{c}{$\begin{array}{c}\text { SOX9-positive } \\
\text { foci, } \mathrm{n}(\%)\end{array}$} \\
\cline { 3 - 4 } Lesion & No. of foci & Nuclear & Cytoplasmic \\
\hline Normal epithelia & 29 & $16(55)$ & $0(0)$ \\
Epithelial dysplasia & 48 & $38(79)$ & $0(0)$ \\
CIS & 41 & $38(93)$ & $0(0)$ \\
SCC & 49 & $43(88)$ & $43(88)$ \\
\hline
\end{tabular}

SOX9, SRY-box 9; SCC, squamous cell carcinoma; CIS, carcinoma in situ.

Statistical analysis. The association between SOX9 expression profiles and the patient characteristics were studied using Chi-squared test $(n=49)$. Survival curves (disease-specific survival and relapse-free survival) were plotted using the Kaplan-Meier method ( $\mathrm{n}=49$ ), and statistical differences were compared using the Wilcoxon test $(n=49)$. A P-value of $<0.05$ was considered statistically significant. Differences in cell growth and migration between the HSC-3 and HSC-4 cells were assessed by the Student's t-test (growth, $n=6$; migration, n=4). All statistical analyses were performed using XLSTAT-Premium (Addinsoft Corp., New York, NY, USA).

\section{Results}

SOX9 expression profiles in oral epithelial lesions. Epithelial dysplasia, CIS and SCC were differentiated according to the expression of perlecan (24), Ki-67 (25) and K17 (26). The tissue specimens from the 49 patients contained 29 foci of normal epithelia, 48 foci of epithelial dysplasia and 41 foci of CIS (Table I). In normal epithelia (Fig. 1A-E), very weak staining for SOX9 in the nuclei of parabasal cells was detected in 16 of the 29 foci (Fig. 1B), and no signals were detectable in the rest. In epithelial dysplasia with a two-phase appearance (moderate degree) (Fig. 1F-J), SOX9 positivity was detected in the nuclei of the proliferating cells in the lower half (Fig. 1G), which were positive for Ki-67 (Fig. 1I) in 38 of the 48 foci. In differentiated CIS (Fig. 1K-O), which exhibited a significant degree of keratinization toward the surface, Ki-67 positivity was mainly observed in the basal zone, including the first layer of epithelium (Fig. 1N), whereas the upper half layer (nearer the surface) was positive for K17 (Fig. 1O). Nuclear positivity for SOX9 was detected throughout the epithelial layer (Fig. 1L) in 38 of the 41 foci. Perlecan was deposited in the intraepithelial space (Fig. 1M). The number of SOX9-positive cells was significantly higher than the number of Ki-67-positive cells (Fig. 1L and N).

In the invasive nests of SCC (Fig. 1P-T), SOX9 was strongly expressed in SCC cells in 45 of the 49 cases (Fig. 1Q), and perlecan was abundantly expressed in the stromal space around the nests (Fig. 1R). K17 positivity was mainly observed in the centers of SCC nests (Fig. 1T), and was not always coincident with the area demonstrating SOX9-positive cells. The immunolocalization of SOX9 


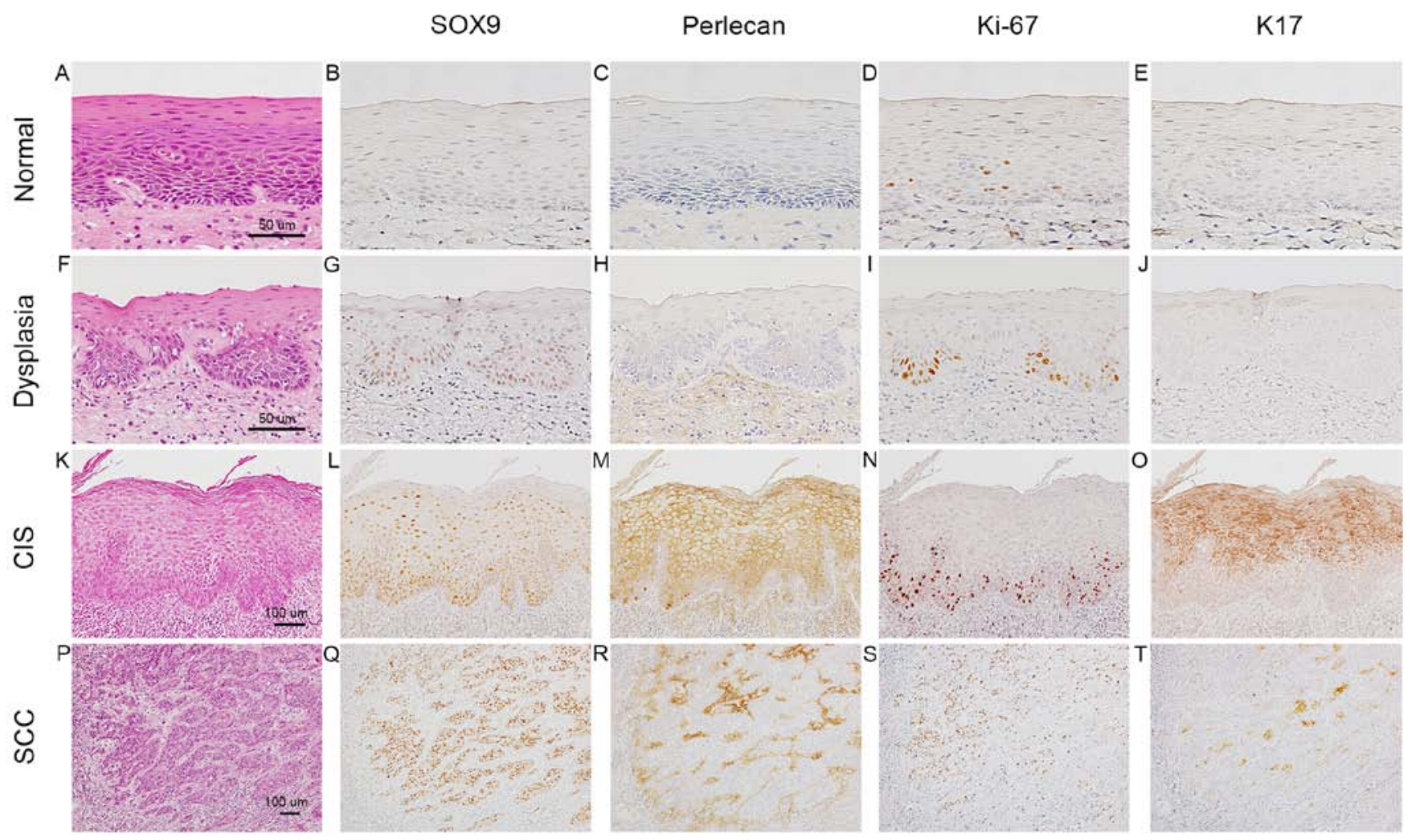

Figure 1. Immunohistochemical expression profiles of SOX9 in normal oral epithelia, epithelial dysplasia, carcinoma in situ (CIS) and squamous cell carcinoma (SCC). Normal epithelia (A-E), epithelial dysplasia (F-J), differentiated CIS (K-O), SCC (P-T). Hematoxylin and eosin (H\&E) staining (A, F, K and P); immunoperoxidase staining for SOX9 (B, G, L and Q), perlecan (C, H, M and R), Ki-67 (D, I, N and S) and keratin (K)17 (E, J, O and T). In normal epithelia (A), SOX9 (B) and perlecan (C) are not evident. In epithelial dysplasia (F), weak SOX9 positivity is observed in the nuclei of the parabasal cells (G), parts of which are also positive for Ki-67 (I). In differentiated CIS (K), the lower half of the epithelial layer is occupied by SOX9-positive cells (L), around which perlecan is intraepithelially deposited (M). In SCC (P), SOX9 expression is enhanced further (Q) and perlecan is diffusely deposited in the cancer stroma (R). SOX9, SRY-box 9.

was observed in the nuclei of the SCC cells in 43 of the 45 cases (Fig. 2A and B). Intriguingly, in 43 of the 45 cases, positive signals for SOX9 were observed in both the cytoplasm and nuclei of SCC cells (Fig. 2C and D); however, cytoplasmic staining was not detectable in epithelial dysplasia and CIS (Table I). In addition, in some subdivisions of the SCC cell areas, SOX9 positivity was found only in the cytoplasm (Fig. 2E and F).

Correlation between SOX9 localization and clinicopathological factors. After determining the proportions of the nuclear and cytoplasmic SOX9-positive areas to the entire area of the SCC, 49 tumors were categorized into two groups using a cut-off point of 25\% SOX9 positivity for both nuclear and cytoplasmic proportions. Consequently, 39 tumors $(80 \%)$ were categorized as having high nuclear expression and 35 tumors (71\%) were categorized as exhibiting high cytoplasmic expression. The correlation of high nuclear and cytoplasmic expression of SOX9 with the clinicopathological factors is summarized in Table II. High nuclear SOX9 expression did not correlate with any clinicopathological factors. However, high cytoplasmic SOX9 expression significantly correlated with regional recurrence $(\mathrm{P}=0.026)$ and female gender $(\mathrm{P}=0.037)$, although both nuclear and cytoplasmic SOX9 expression did not correlate with the pathological $\mathrm{T}$ factor, clinical stage or histologic grade.
Additionally, we examined the metastatic cervical lymph nodes in 12 of the 14 cases with high cytoplasmic SOX 9 expression in the primary lesion. Similar to the primary lesions (Fig. 2G), the metastatic SCC cells demonstrated cytoplasmic SOX9 expression (Fig. 2H).

Cytoplasmic SOX9 positivity is significantly correlated with poor clinical outcomes. High nuclear SOX9 expression was not significantly correlated with disease-specific survival (Fig. 3A, $\mathrm{P}=0.715$ ) or relapse-free survival (Fig. 3B, $\mathrm{P}=0.588$ ). However, high cytoplasmic SOX9 expression was significantly correlated with both shorter disease-specific survival (Fig. 3C, $\mathrm{P}=0.044$ ) and relapse-free survival (Fig. 3D, $\mathrm{P}=0.040)$.

Cytoplasmic expression of SOX9 is correlated with higher cell migration ability. Immunofluoresence staining revealed that SOX9 was localized in both the nuclei and cytoplasm of HSC-3 cells (Fig. 4A). In contrast, the localization of SOX9 was limited to the nuclei in HSC-4 cells (Fig. 4B). Both mRNA and protein expression levels of SOX9 were much higher in HSC-3 cells than these levels in HSC-4 cells (Fig. 4C and D). Although there was no significant difference in cell growth (Fig. 4E), migration ability was higher in HSC-3 cells than that in HSC-4 cells (Fig. 4F). Taken together, cytoplasmic SOX9 expression in HSC-3 cells might be related to the higher migration ability of HSC-3 cells. 

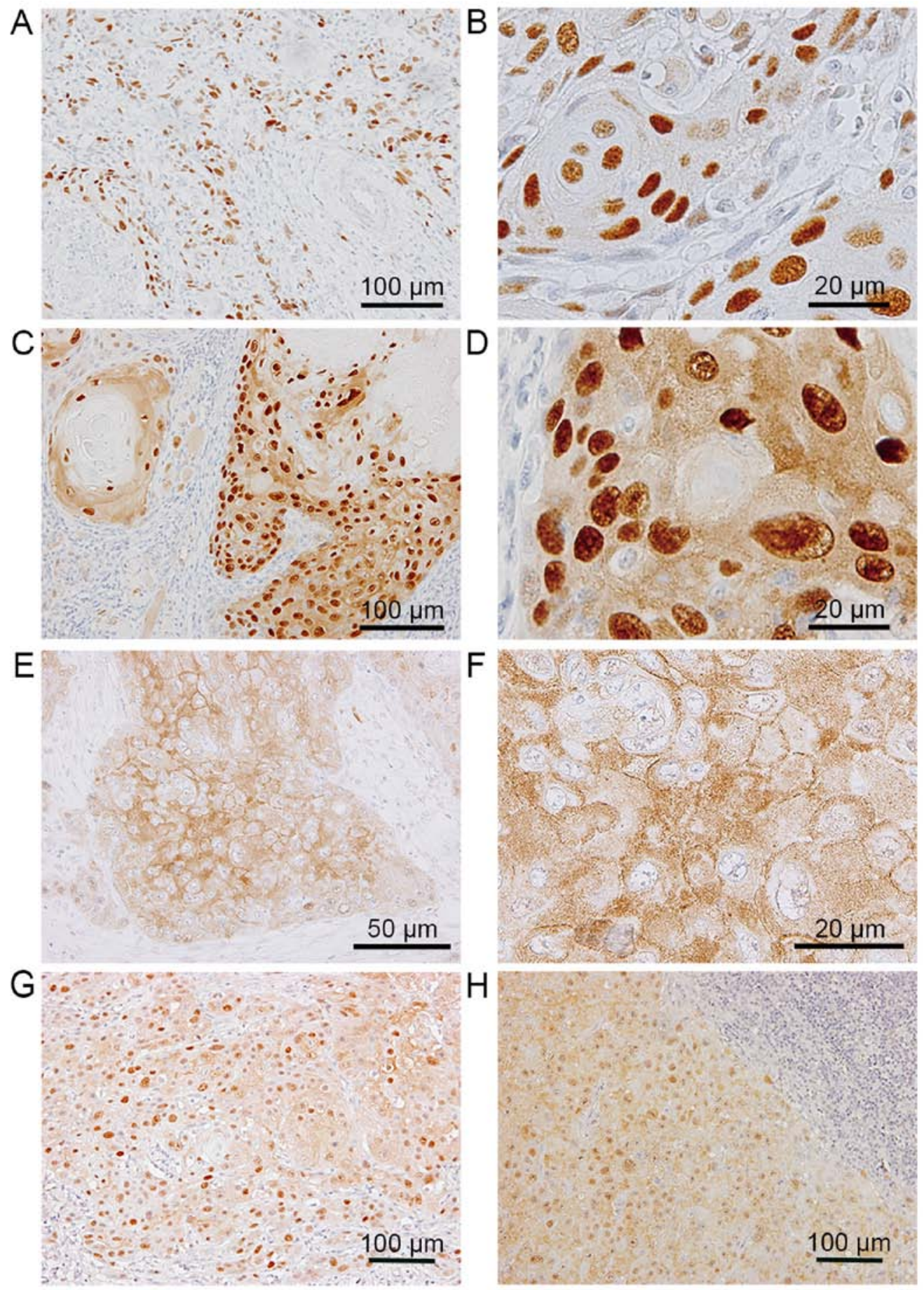

Figure 2. Localization patterns of SOX9 in squamous cell carcinoma (SCC) cells in primary and metastatic lesions. In the primary lesions, the localization of SOX9 was found only in the nuclei (A and B), both in the nuclei and the cytoplasm (C and D), or only in the cytoplasm (E and F). Cytoplasmic SOX9 was detected in both the primary lesion $(\mathrm{G})$ and the metastatic lymph nodes $(\mathrm{H})$ resected from the same patient. SOX9, SRY-box 9.

\section{Discussion}

In the present study, we demonstrated for the first time the elevated expression of SOX9 in oral epithelial lesions, including epithelial dysplasia, CIS and SCC through immunohistochemical analysis and posited that the cytoplasmic expression of SOX9 in SCC cells is a potential prognostic factor for OSCC.

We successfully demonstrated that SOX9 expression was elevated in a stepwise manner in epithelial dysplasia, CIS and SCC. However, only weak signals were detected in the parabasal cells of the normal epithelia (Fig. 1). Similar to our results, several studies have reported that compared with non-neoplastic cells in various organs, cancer cells express SOX9 at higher levels $(6,7,11)$. In epithelial dysplasia and CIS, SOX9-positive cells were distributed in the layer farther from the surface, whereas the number of Ki-67-positive proliferating cells was higher; these data suggest that SOX9 expression is positively correlated with cell proliferation during carcinogenesis. The reduction of endogenous SOX9 
Table II. Relationship between clinicopathological factors of the OSCC cases and SOX9 expression.

\begin{tabular}{|c|c|c|c|c|c|c|c|}
\hline \multirow[b]{2}{*}{ Clinicopathological factors } & \multirow[b]{2}{*}{$\mathrm{n}$} & \multicolumn{3}{|c|}{ Nuclear SOX9 } & \multicolumn{3}{|c|}{ Cytoplasmic SOX9 } \\
\hline & & High & Low & P-value & High & Low & P-value \\
\hline \multicolumn{8}{|l|}{ Age (years) } \\
\hline$<$ Median & 24 & 20 & 4 & 0.524 & 16 & 8 & 0.470 \\
\hline$\geq$ Median & 25 & 19 & 6 & & 19 & 6 & \\
\hline \multicolumn{8}{|l|}{ Sex } \\
\hline Male & 27 & 21 & 6 & 0.727 & 16 & 11 & 0.037 \\
\hline Female & 22 & 18 & 4 & & 19 & 3 & \\
\hline \multicolumn{8}{|l|}{ Tumor site } \\
\hline Tongue & 28 & 21 & 7 & 0.796 & 22 & 6 & 0.246 \\
\hline Buccal mucosa & 5 & 5 & 0 & & 3 & 2 & \\
\hline Oral floor & 5 & 4 & 1 & & 2 & 3 & \\
\hline Gingiva & 7 & 6 & 1 & & 6 & 1 & \\
\hline Palate & 1 & 1 & 0 & & 1 & 0 & \\
\hline Other & 3 & 2 & 1 & & 1 & 2 & \\
\hline \multicolumn{8}{|l|}{ Pathological T factor } \\
\hline $\mathrm{T} 1$ & 17 & 15 & 2 & 0.567 & 12 & 5 & 0.827 \\
\hline $\mathrm{T} 2$ & 26 & 19 & 7 & & 18 & 8 & \\
\hline $\mathrm{T} 3$ & 2 & 2 & 0 & & 2 & 0 & \\
\hline $\mathrm{T} 4$ & 4 & 3 & 1 & & 3 & 1 & \\
\hline \multicolumn{8}{|l|}{ Lymph node metastasis } \\
\hline Absent & 38 & 31 & 7 & 0.521 & 27 & 11 & 0.914 \\
\hline Present & 11 & 8 & 3 & & 8 & 3 & \\
\hline \multicolumn{8}{|l|}{ Clinical stage } \\
\hline I & 17 & 15 & 2 & 0.388 & 12 & 5 & 0.561 \\
\hline II & 23 & 16 & 7 & & 15 & 8 & \\
\hline III & 3 & 3 & 0 & & 3 & 0 & \\
\hline IV & 6 & 5 & 1 & & 5 & 1 & \\
\hline \multicolumn{8}{|l|}{ Histological grade } \\
\hline G1 & 42 & 32 & 10 & 0.148 & 30 & 12 & 0.694 \\
\hline $\mathrm{G} 2+\mathrm{G} 3$ & 7 & 7 & 0 & & 5 & 2 & \\
\hline \multicolumn{8}{|l|}{ Local recurrence } \\
\hline Absent & 40 & 33 & 7 & 0.287 & 29 & 11 & 0.726 \\
\hline Present & 9 & 6 & 3 & & 6 & 3 & \\
\hline \multicolumn{8}{|l|}{ Regional recurrence } \\
\hline Absent & 30 & 24 & 6 & 0.929 & 18 & 12 & 0.026 \\
\hline Present & 19 & 15 & 4 & & 17 & 2 & \\
\hline \multicolumn{8}{|l|}{ Late distant metastasis } \\
\hline Absent & 36 & 30 & 6 & 0.280 & 23 & 13 & 0.052 \\
\hline Present & 13 & 9 & 4 & & 12 & 1 & \\
\hline
\end{tabular}

SOX9, SRY-box 9; OSCC, oral squamous cell carcinoma.

expression by RNA interference together with the increased expression of the cell cycle inhibitor p27 in prostatic cancer cell lines has been shown to result in growth arrest (7). Meanwhile, the knockdown of SOX9 along with the upregulation of p21 in lung adenocarcinoma cell lines has been reported to inhibit cell growth (27). Conversely, in esophageal SCC cells, SOX9 overexpression has been shown to induce cell proliferation and tumorigenicity through the activation of PI3K/Akt signaling (11). Besides, SOX9 expression is regulated by the Wnt signaling pathway (7). Consistent with our previous report, the nuclear transition of $\beta$-catenin was observed in the lower layer of epithelial dysplasia (28), suggesting that the upregulation of SOX9 expression in oral mucosal epithelial cells may be controlled by the Wnt pathway. 
A Disease-specific survival: nuclear sox9

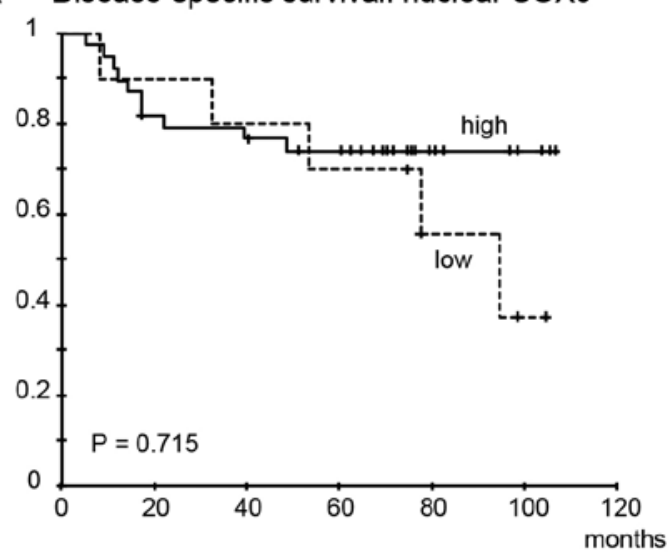

C Disease-specific survival: cytoplasmic SOX9

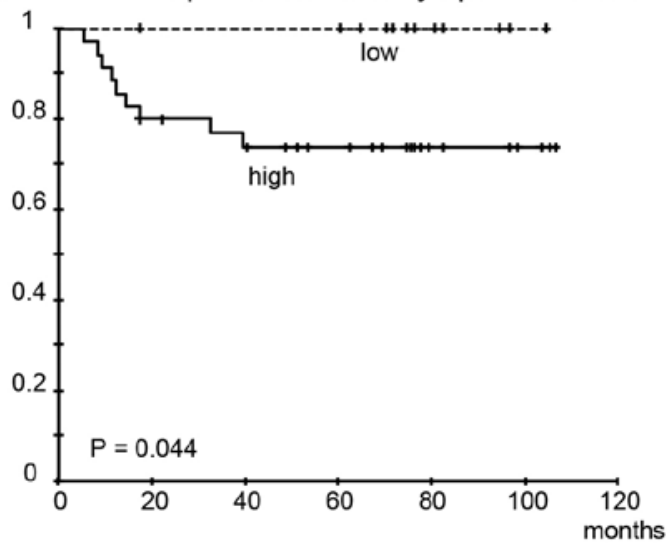

B Relapse-free survival: nuclear Sox9$$
1
$$

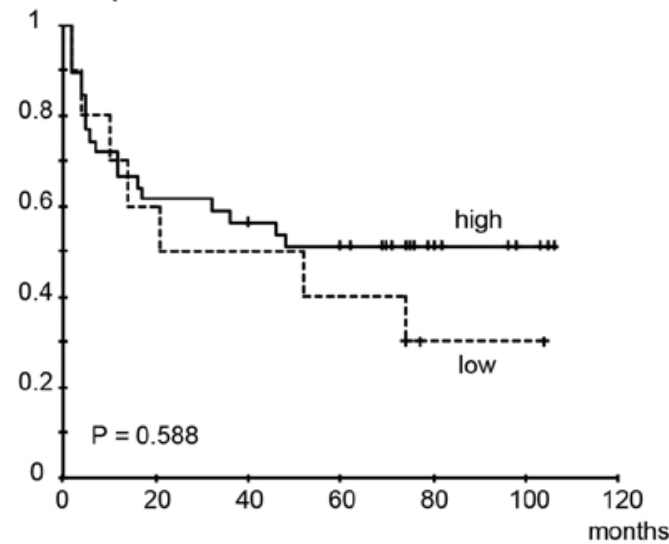

D Relapse-free survival: cytoplasmic SOX9

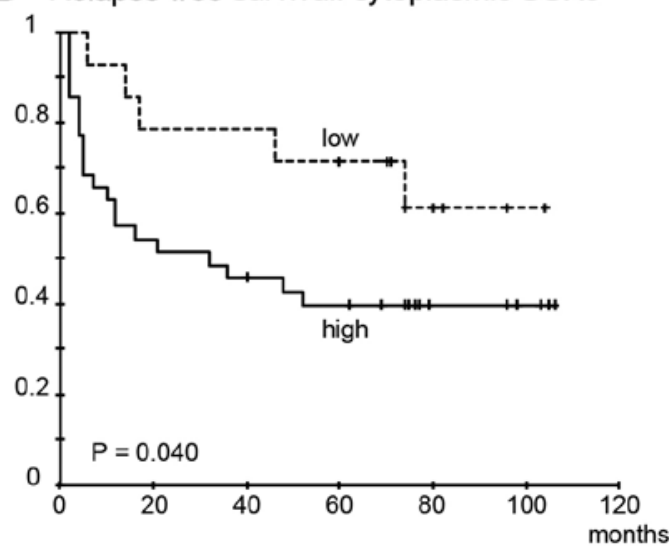

Figure 3. Kaplan-Meier curves showing disease-specific survival and relapse-free survival of the patients with OSCC according to SOX9 expression status. High nuclear SOX9 expression was not associated with disease-specific survival $(\mathrm{A}, \mathrm{P}=0.715)$ or relapse-free survival $(\mathrm{B}, \mathrm{P}=0.588)$. High cytoplasmic SOX9 expression was found to be significantly correlated with shorter disease-specific survival $(\mathrm{C}, \mathrm{P}=0.044)$ and relapse-free survival $(\mathrm{D}, \mathrm{P}=0.040)$. $\mathrm{SOX}$, SRY-box 9; OSCC, oral squamous cell carcinoma.

The present study revealed that SOX9 expression was localized in the cytoplasm as well as nuclei of SCC cells but not of the cells in epithelial dysplasia and CIS (Fig. 1 and Table I). Chakravarty et al reported that in the invasive ductal carcinoma of the breast, SOX9 accumulation in the cytoplasm was correlated with enhanced cell proliferation (8). We speculate that the elevated SOX9 expression in this study resulted in its accumulation in the cytoplasm given the fact that cytoplasmic SOX9 was not detected in HSC-4 cells, which only weakly expressed SOX9, but was detected in HSC-3 cells, which showed strongly expressed SOX9 (Fig. 4). Malki et al demonstrated the retention of synthesized SOX9 in the cytoplasm via interaction with the microtubule network in male pre-Sertoli cells (29). Cytoplasmic SOX9 expression in SCC cells may be induced through a similar mechanism. However, more detailed investigations should be conducted since cytoplasmic protein localization also results from post-translational modifications, such as acetylation $(30,31)$.

Furthermore, we demonstrated that cytoplasmic SOX9 expression in a higher proportion of SCC cells was significantly correlated with the late occurrence of lymph node metastasis and shorter relapse-free and disease-specific survival (Table II and Fig. 3); these data indicate that SOX9 could be a useful prognostic marker for OSCC, similar to its use in various types of human cancers $(8,9,11)$. SOX9 orchestrates the cellular dynamics that involve migration, invasion and metastasis in addition to cell proliferation (18). Larsimont et al demonstrated that SOX9 was found to positively regulate the expression of several ECM components (collagens and laminins), cell adhesion (integrins), and key regulators of actin cytoskeleton dynamics (gelsolin and palladin) in mouse models of cutaneous basal cell carcinoma (18).

Moreover, SOX9 is known to promote epithelial-mesenchymal transition (EMT) together with SLUG $(32,33)$, one of the key transcriptional factors in EMT (34). When SOX9 and SLUG were overexpressed in breast cancer cells, they cooperatively facilitated EMT, resulting in elevated tumorigenesis and metastasis (32). In addition, SOX9 and SLUG synergistically activated the expression of tenascin $\mathrm{C}$ and periostin, which are important ECM components in cancer invasion and metastasis (33).

In the present study, we illustrated the elevated deposition of perlecan around SOX9-positive CIS cells and in the stromal spaces in SCC (Fig. 1). HSPG2, which encodes perlecan, is one of the key target genes of SOX9 (18). In contrast, perlecan is required for the induction of SOX9 expression in chondrogenic differentiation (35). Therefore, a positive feedback loop between SOX9 and perlecan appears to act in cancer progression. Regarding K17, which is highly expressed in oral epithelial malignancies (26) and 
A
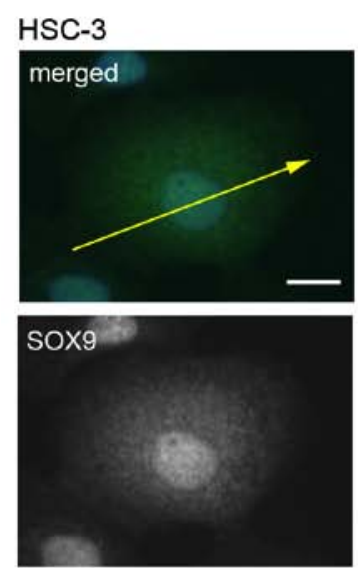

C

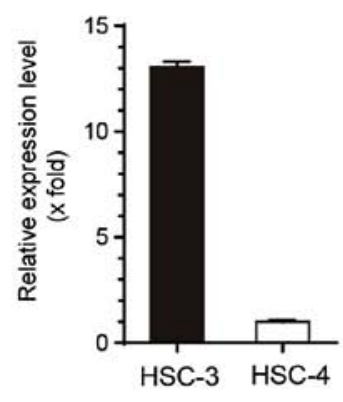

B
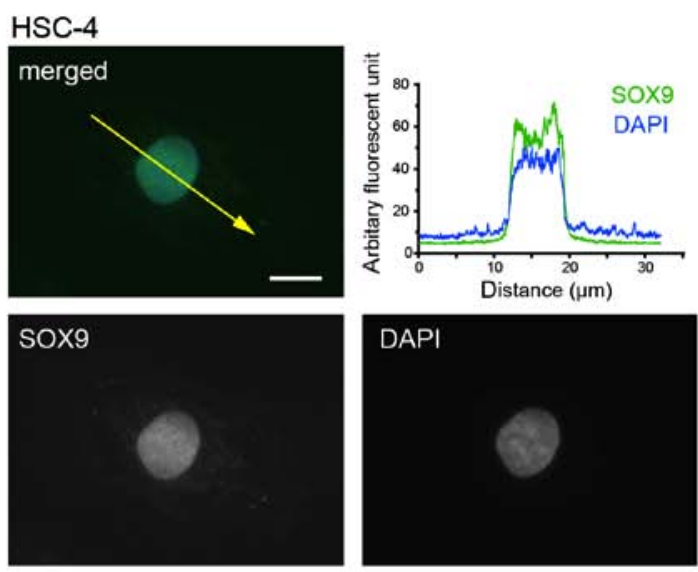

E

$\mathbf{F}$
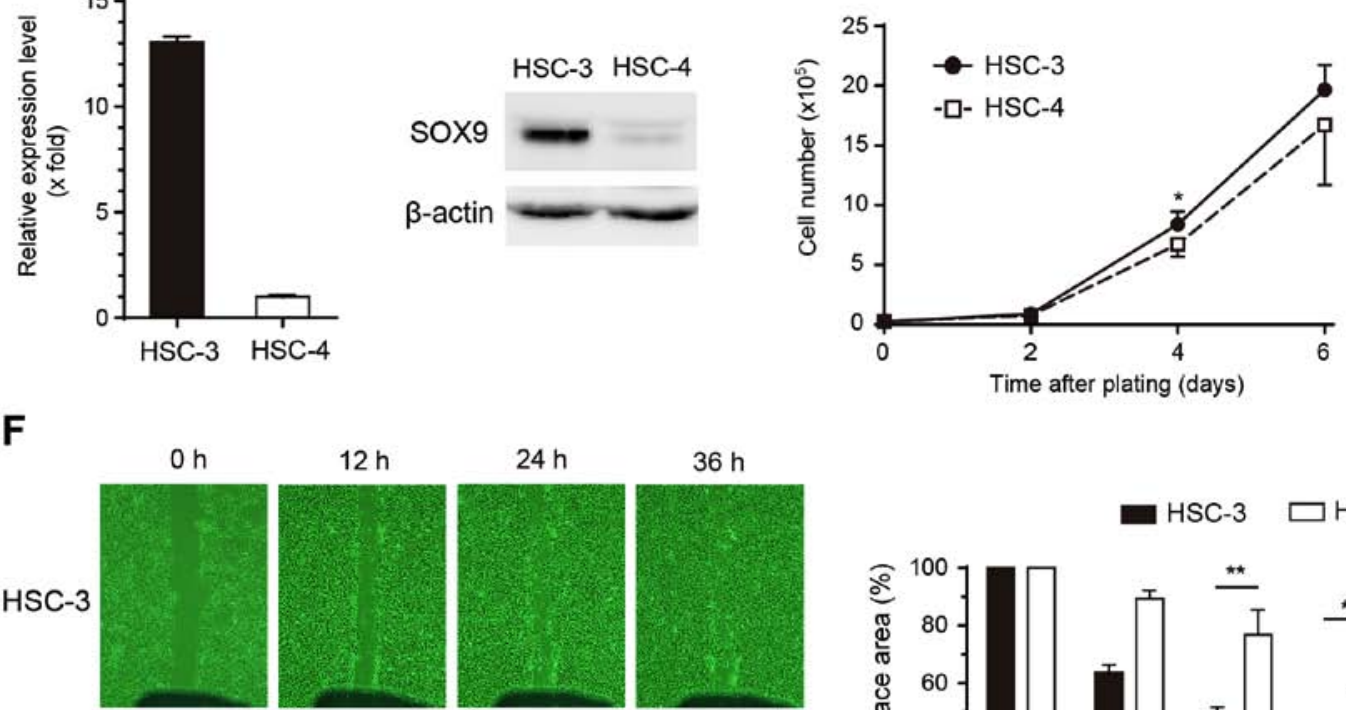

$12 \mathrm{~h}$
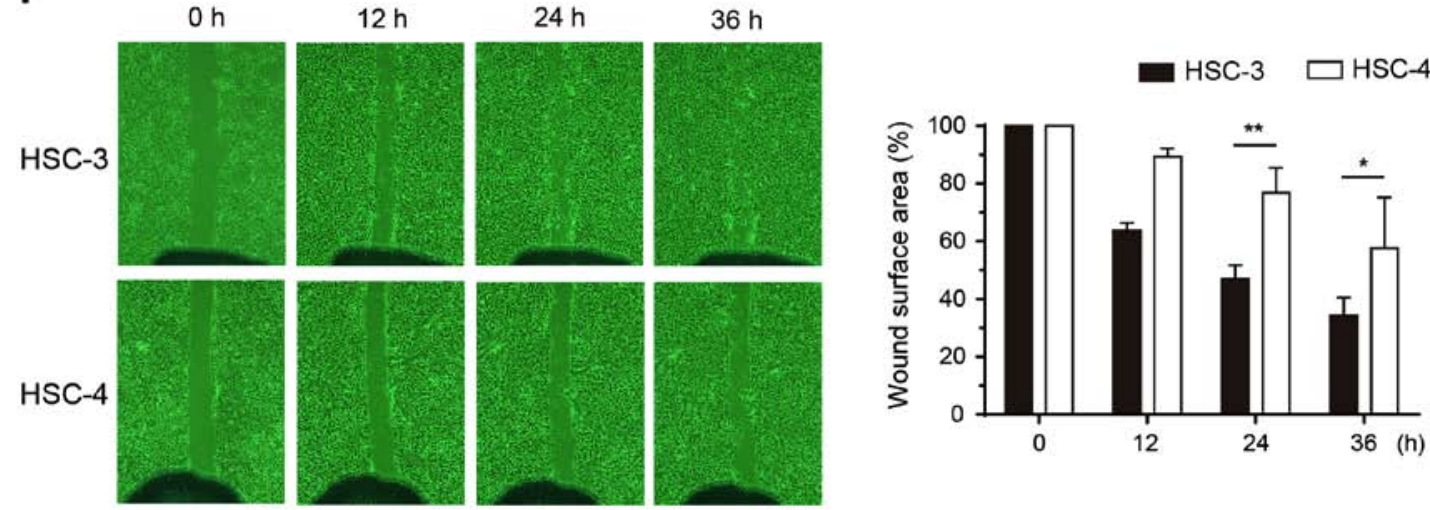

Figure 4. SOX9 expression profiles and cellular activities in OSCC cell lines. Immunofluorescent staining for SOX9 in HSC-3 (A) and HSC-4 cells (B). Green, SOX9; blue, DAPI. Scale bars, $10 \mu \mathrm{m}$. The diagrams display the localization of the fluorescent signals in arbitrary fluorescent units. Yellow arrows in the merged image indicate localization in the cross-section corresponding to the fluorescent diagrams. (C) mRNA expression levels by quantitative RT-PCR. (D) Protein expression by western blotting in the HSC-3 and HSC-4 cells. (E) Growth curves of the HSC-3 (closed circles) and HSC-4 (open squares) cell lines. (F) Scratch wound-healing assay. The graph indicates the percentages of wound surface area for the HSC-3 (closed bars) and HSC-4 (open bars) cell lines. ${ }^{*} \mathrm{P}<0.05,{ }^{* *} \mathrm{P}<0.01$. HSC -3 cells show abundant cytoplasmic SOX9 expression compared with HSC-4 cells (A and B). mRNA and protein expression levels are much higher in the HSC-3 cells than in the HSC-4 cells (C and D). There is no definite difference in cell growth between the two cell lines (E). The cell migratory ability of HSC-3 is significantly higher than that of HSC-4 (F). SOX9, SRY-box 9; OSCC, oral squamous cell carcinoma.

promotes cell proliferation and migration (36), we observed no evidence of colocalization with SOX9. Further investigation is warranted to determine the potential role of SOX9 in regulating K17 expression.

In conclusion, SOX 9 may play important roles in the carcinogenesis and progression of OSCC. SOX9 expression represents a potential marker for predicting poor prognosis in patients with OSCC. However, future studies are warranted to elucidate the precise mechanisms underlying the cytoplasmic localization of SOX9 expression.

\section{Acknowledgements}

The authors would like to thank Ms. Shizuko Horiuchi and Ms. Yuki Takahashi for assistance in the tissue sample preparation.

\section{Funding}

The present study was supported in part by a Grant-in Aid for Scientific Research from the Japan Society for the Promotion 
of Science (JSPS KAKENHI grant no. 15K11006 to MY and JSPS KAKENHI (grant no. 15K11069 to SM).

\section{Availability of data and materials}

This research's data is on 'figshere'. (https://figshare. com/projects/Cytoplasmic_expression_of_SOX9_as_a_poor_ prognostic_factor_for_oral_squamous_cell_carcinoma_/30118).

\section{Author's contributions}

YS, MY, SM, RT and JIT conceived and designed the study. YS, MY, SM, JC and TA performed the experiments. YS and MY wrote the paper. SM, JC, RT and JIT reviewed and edited the manuscript. All authors read and approved the manuscript and agree to be accountable for all aspects of the research in ensuring that the accuracy or integrity of any part of the work are appropriately investigated and resolved.

\section{Ethics approval and consent to participate}

The present study was certified by the Ethics Committee of Niigata University. The Ethics Board of the Niigata University Graduate School of Medicine and Dental Sciences (Oral Life Science) reviewed and approved the experimental protocol for analyzing the surgical materials (approval no. 12-10-13).

\section{Patient consent for publication}

Written informed consent was obtained from all the patients.

\section{Competing interests}

The authors state that they have no competing interests.

\section{References}

1. Jo A, Denduluri S, Zhang B, Wang Z, Yin L, Yan Z, Kang R, Shi LL, Mok J, Lee MJ and Haydon RC: The versatile functions of Sox 9 in development, stem cells, and human diseases. Genes Dis 1: 149-161, 2014.

2. Pritchett J, Athwal V, Roberts N, Hanley NA and Hanley KP: Understanding the role of SOX9 in acquired diseases: Lessons from development. Trends Mol Med 17: 166-174, 2011.

3. Harley VR, Clarkson MJ and Argentaro A: The molecular action and regulation of the testis-determining factors, SRY (sex-determining region on the Y chromosome) and SOX9 [SRY-related high-mobility group (HMG) box 9]. Endocr Rev 24: 466-487, 2003.

4. Akiyama $\mathrm{H}$ : Control of chondrogenesis by the transcription factor Sox9. Mod Rheumatol 18: 213-219, 2008.

5. Vidal VP, Chaboissier MC, Lutzkendorf S, Cotsarelis G, Mill P, Hui CC, Ortonne N, Ortonne JP and Schedl A: Sox9 is essential for outer root sheath differentiation and the formation of the hair stem cell compartment. Curr Biol 15: 1340-1351, 2005.

6. Shi G, Sohn KC, Li Z, Choi DK, Park YM, Kim JH, Fan YM, $\mathrm{Nam}$ YH, Kim S, Im M, et al: Expression and functional role of Sox9 in human epidermal keratinocytes. PLoS One 8: e54355, 2013.

7. Wang H, McKnight NC, Zhang T, Lu ML, Balk SP and Yuan X: SOX9 is expressed in normal prostate basal cells and regulates androgen receptor expression in prostate cancer cells. Cancer Res 67: 528-536, 2007.

8. Chakravarty G, Moroz K, Makridakis NM, Lloyd SA, Galvez SE, Canavello PR, Lacey MR, Agrawal K and Mondal D: Prognostic significance of cytoplasmic SOX9 in invasive ductal carcinoma and metastatic breast cancer. Exp Biol Med 236: 145-155, 2011.
9. Lü B, Fang Y, Xu J, Wang L, Xu F, Xu E, Huang Q and Lai M: Analysis of SOX9 expression in colorectal cancer. Am J Clin Pathol 130: 897-904, 2008.

10. Vidal VP, Ortonne N and Schedl A: SOX9 expression is a general marker of basal cell carcinoma and adnexal-related neoplasms. J Cutan Pathol 35: 373-379, 2008.

11. Hong Y, Chen W, Du X, Ning H, Chen H, Shi R, Lin S, Xu R, $\mathrm{Zhu} \mathrm{J}, \mathrm{Wu} \mathrm{S}$, et al: Upregulation of sex-determining region Y-box 9 (SOX9) promotes cell proliferation and tumorigenicity in esophageal squamous cell carcinoma. Oncotarget 6: 31241-31254, 2015 .

12. Ikarashi T, Ida-Yonemochi H, Ohshiro K, Cheng $\mathrm{J}$ and Saku T: Intraepithelial expression of perlecan, a basement membrane-type heparan sulfate proteoglycan reflects dysplastic changes of the oral mucosal epithelium. J Oral Pathol Med 33: 87-95, 2004.

13. Maruyama S, Shimazu Y, Kudo T, Sato K, Yamazaki M, Abé T, Babkair H, Cheng J, Aoba T and Saku T: Three-dimensional visualization of perlecan-rich neoplastic stroma induced concurrently with the invasion of oral squamous cell carcinoma. J Oral Pathol Med 43: 627-636, 2014.

14. Maruyama S, Cheng J, Yamazaki M, Liu A and Saku T: Keratinocyte growth factor colocalized with perlecan at the site of capsular invasion and vascular involvement in salivary pleomorphic adenomas. J Oral Pathol Med 38: 377-385, 2009.

15. Toyoshima K, Kimura S, Cheng J, Oda Y, Mori KJ and Saku T: High-molecular-weight fibronectin synthesized by adenoid cystic carcinoma cells of salivary gland origin. Jpn J Cancer Res 90: 308-319, 1999.

16. Hasegawa M, Cheng J, Maruyama S, Yamazaki M, Abé T, Babkair H, Saito C and Saku T: Differential immunohistochemical expression profiles of perlecan-binding growth factors in epithelial dysplasia, carcinoma in situ, and squamous cell carcinoma of the oral mucosa. Pathol Res Pract 212: 426-436, 2016.

17. Warren CR, Grindel BJ, Francis L, Carson DD and Farach-Carson MC: Transcriptional activation by NFkappaB increases perlecan/HSPG2 expression in the desmoplastic prostate tumor microenvironment. J Cell Biochem 115: 1322-1333, 2014.

18. Larsimont JC, Youssef KK, Sánchez-Danés A, Sukumaran V, Defrance M, Delatte B, Liagre M, Baatsen P, Marine JC, Lippens S, et al: Sox9 controls self-renewal of oncogene targeted cells and links tumor initiation and invasion. Cell Stem Cell 17: 60-73, 2015.

19. UICC International Union Against Cancer: TNM Classification of Malignant Tumours. Sobin LH, Gospodarowicz MK and Wittekind C (eds). 7th edition. Wiley-Blackwell, Hoboken, NJ, pp25-28, 2009.

20. Saku T and Furthmayr H: Characterization of the major heparan sulfate proteoglycan secreted by bovine aortic endothelial cells in culture. Homology to the large molecular weight molecule of basement membranes. J Biol Chem 264: 3514-3523, 1989.

21. Momose F, Araida T, Negishi A, Ichijo H, Shioda S and Sasaki S: Variant sublines with different metastatic potentials selected in nude mice from human oral squamous cell carcinomas. J Oral Pathol Med 18: 391-395, 1989.

22. Livak KJ and Schmittgen TD: Analysis of relative gene expression data using real-time quantitative PCR and the $2^{-\Delta \Delta C \mathrm{~T}}$ method. Methods 25: 402-408, 2001.

23. Yamazaki M, Maruyama S, Abé T, Essa A, Babkair H, Cheng J and Saku T: MFG-E8 expression for progression of oral squamous cell carcinoma and for self-clearance of apoptotic cells. Lab Invest 94: 1260-1272, 2014.

24. Ahsan MS, Yamazaki M, Maruyama S, Kobayashi T, Ida-Yonemochi $\mathrm{H}$, Hasegawa M, Henry Ademola A, Cheng J and Saku T: Differential expression of perlecan receptors, $\alpha$-dystroglycan and integrin $\beta 1$, before and after invasion of oral squamous cell carcinoma. J Oral Pathol Med 40: 552-559, 2011.

25. Kobayashi T, Maruyama S, Cheng J, Ida-Yonemochi H, Yagi M, Takagi R and Saku T: Histopathological varieties of oral carcinoma in situ: Diagnosis aided by immunohistochemistry dealing with the second basal cell layer as the proliferating center of oral mucosal epithelia. Pathol Int 60: 156-166, 2010.

26. Mikami T, Cheng J, Maruyama S, Kobayashi T, Funayama A, Yamazaki M, Adeola HA, Wu L, Shingaki S, Saito C and Saku T: Emergence of keratin 17 vs. loss of keratin 13: Their reciprocal immunohistochemical profiles in oral carcinoma in situ. Oral Oncol 47: 497-503, 2011. 
27. Jiang SS, Fang WT, Hou YH, Huang SF, Yen BL, Chang JL, Li SM, Liu HP, Liu YL, Huang CT, et al: Upregulation of SOX9 in lung adenocarcinoma and its involvement in the regulation of cell growth and tumorigenicity. Clin Cancer Res 16: 4363-4373, 2010 .

28. Alvarado CG, Maruyama S, Cheng J, Ida-Yonemochi H, Kobayashi T, Yamazaki M, Takagi R and Saku T: Nuclear translocation of $\beta$-catenin synchronized with loss of E-cadherin in oral epithelial dysplasia with a characteristic two-phase appearance. Histopathology 59: 283-291, 2011.

29. Malki S, Berta P, Poulat F and Boizet-Bonhoure B: Cytoplasmic retention of the sex-determining factor SOX9 via the microtubule network. Exp Cell Res 309: 468-475, 2005.

30. Bar Oz M, Kumar A, Elayyan J, Reich E, Binyamin M, Kandel L, Liebergall M, Steinmeyer J, Lefebvre V and Dvir-Ginzberg M: Acetylation reduces SOX9 nuclear entry and ACAN gene transactivation in human chondrocytes. Aging Cell 15: 499-508, 2016.

31. Sim H, Argentaro A and Harley VR: Boys, girls and shuttling of SRY and SOX9. Trends Endocrinol Metab 19: 213-222, 2008.

32. Guo W, Keckesova Z, Donaher JL, Shibue T, Tischler V, Reinhardt F, Itzkovitz S, Noske A, Zürrer-Härdi U, Bell G, et al: Slug and Sox 9 cooperatively determine the mammary stem cell state. Cell 148: 1015-1028, 2012.
33. Fazilaty H, Gardaneh M, Akbari P, Zekri A and Behnam B: SLUG and SOX9 cooperatively regulate tumor initiating niche factors in breast cancer. Cancer Microenviron 9: 71-74, 2016.

34. Savagner P, Yamada KM and Thiery JP: The zinc-finger protein slug causes desmosome dissociation, an initial and necessary step for growth factor-induced epithelial-mesenchymal transition. J Cell Biol 137: 1403-1419, 1997.

35. Sadatsuki R, Kaneko H, Kinoshita M, Futami I, Nonaka R, Culley KL, Otero M, Hada S, Goldring MB, Yamada Y, et al: Perlecan is required for the chondrogenic differentiation of synovial mesenchymal cells through regulation of Sox 9 gene expression. J Orthop Res 35: 837-846, 2017.

36. Khanom R, Nguyen CT, Kayamori K, Zhao X, Morita K, Miki Y, Katsube K, Yamaguchi A and Sakamoto K: Keratin 17 is induced in oral cancer and facilitates tumor growth. PLoS One 11: e0161163, 2016.

This work is licensed under a Creative Commons Attribution-NonCommercial-NoDerivatives 4.0 International (CC BY-NC-ND 4.0) License. 